\title{
Voltage Transformer Fault Detection by Wire-Breaking Recognition
}

\author{
Yuqin $\mathrm{YaO}^{1, a}$, Yuxin $\mathrm{Mo}^{1}$, Qiushi Liu ${ }^{1}$ \\ ${ }^{1}$ Chengdu University of Information Technology, Chengdu, 610225, China \\ ${ }^{a}$ email: yyq@cuit.edu.cn
}

Key Words: Voltage Transformer; Wire Breaking; Simulation

\begin{abstract}
Voltage transformer may appear disconnection fault at operation time. This will greatly affect the protection devices work properly. Thus the impact of this failure, as well as an accurate method of determining the fault is worth studied. In this paper, based on the actual grid model, the specific effects caused by the fault were analyzed principally. And whether the general methods could identify the fault in several conditions is simulated.
\end{abstract}

\section{Overview}

Voltage transformer obtains the grid voltage for protection devices. It transfers high grid voltage to standard voltage $(100 \mathrm{~V})$. All instruments and protection devices which are input voltage, including transformer main protecting and backup protection, will be affected by breaking fault ${ }^{[1]}$.

For the fault, on time identification is decided whether relay could work correctly. Due to the special nature of the power supply system, the conventional voltage transformer breaking criterion required to undergo verification analysis of certain special conditions, in order to ensure its applicability ${ }^{[2]}$.

\section{Connection Analysis of PT in Grid}

General connection method is shown in Fig.1.

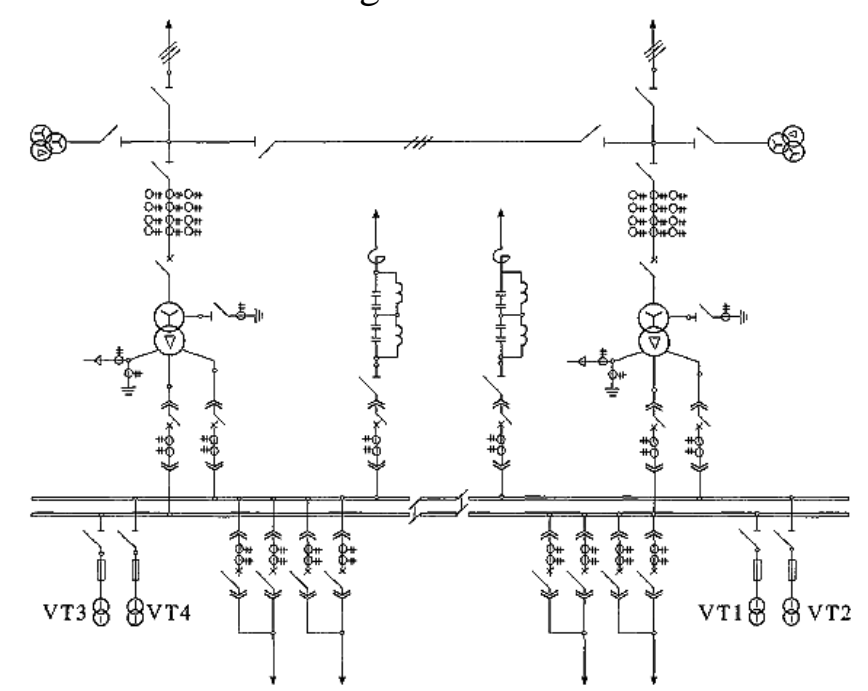

Fig.1 main connection of substation

Whether voltage transformer is disconnected in power system is generally judged based on the positive or negative-sequence voltage ${ }^{[3]}$. For example, the fault identification method of NARI is:

(1) Positive sequence voltage is less than $30 \mathrm{~V}$, and any one phase current is greater than $0.04 I_{n}$ ( $I_{n}$ is the rated current) or switches together bits;

(2) Negative sequence voltage is greater than $8 \mathrm{~V}$.

Three-phase power supply system - two-phase system, the load is single phase load, and thus the low pressure side of the power supply system with only two single-phase voltage transformer, which determines the criterion can not use the low voltage side of the transformer power system to 
identify voltage transformer off line ${ }^{[4]}$. Load also has a non-continuous, asymmetry and other characteristics, the high side voltage transformers are often able to measure a large negative sequence component, which generally do not have high side transformer criterion to identify the power system voltage transformer disconnected.

\section{Breaking Fault Analysis of Secondly PT}

After the break voltage transformer failure, due to wiring or other reasons, the protection device may be measured to a certain voltage value ${ }^{[5]}$.

(1) Effect of "Three Instruments Method" measurement of PT breaking fault

Low-side voltage transformer substation wiring is shown in Figure 2.

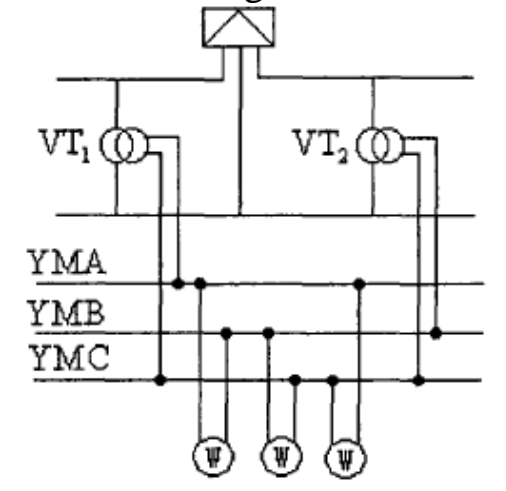

Fig.2 connection of secondly PT

Two power substations were connected to a single arm phase voltage transformer $\mathrm{VT}_{1}$ and $\mathrm{VT}_{2}$ two secondary side voltage transformers are connected to a small high-side bus YMA, on YMB, its low-end small bus together received YMC. Substation generally use the "Three Instrument Method" low-voltage side reactive power measurement, three single-phase meter were received on two of three small bus. The protection device is a voltage measured YMA, YMC or between YMB, the voltage between the YMC.

As can be seen from Figure 2, $\mathrm{VT}_{1}$ happen if the primary or secondary side disconnection fault, because the effect of the partial pressure measurement meter internal resistance, protection devices can still measure the voltage to around $50 \% \mathrm{VT}_{2}$ amount. In fact, due to the presence of other measuring circuit, $\mathrm{VT}_{1}$ break after a failure occurs, the measured voltage of the device to protect the scene about $45 \%$ the amount of voltage. Conversely, if the disconnection occurs, $\mathrm{VT}_{1}$ get fault too.

(2) Impact of the primary voltage transformer fuses for disconnection

The low pressure side of the voltage transformer substation generally by isolating switches and high voltage fuses RD access to high-voltage grid. Voltage transformer high side wiring is shown in Figure 3.

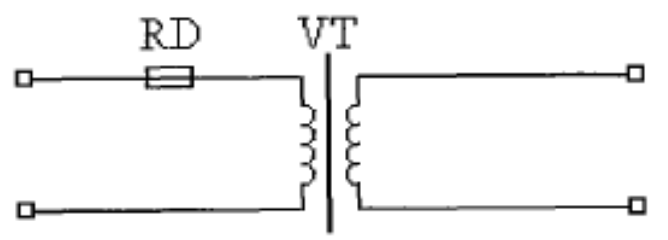

Fig.3 primary connection of PT

High voltage fuse of PT mainly protects inner fault of PT and short circuit fault. Sometimes high-voltage fuse will not completely fuse, then the fuse resistance equivalent to a large resistance. Since the effect of the partial pressure of the fuse, the protection device can be measured to a certain amount of voltage. Field test, when not completely blown fuse, field measurement voltage protection device is about $10 \%$ to $30 \%$ of the actual amount of voltage.

\section{Breaking Fault Simulation}

Some substation in Chengdu-Chongqing line is taken as a simulation example. Power Administration to provide system parameters are: system capacity reference value $S_{j}=100 \mathrm{MVA}$, 
system reference voltage $U_{j}=115 \mathrm{kV}$, maximum operating mode system impedance unit value $\mathrm{Z}_{\text {shigh }}=0.1424$, the minimum operation mode system impedance unit value $\mathrm{Z}_{\text {slow }}^{*}=0.2340$. Transformer YN, dll transformer capacity of 60MVA, rated voltage $110 \mathrm{kV} / 27.5 \mathrm{kV}$, short-circuit voltage percentage was $10.5 \% . \mathrm{K}_{\mathrm{k}}=1.5, \mathrm{U}_{\mathrm{zd}}=5.5 \mathrm{kV}, \mathrm{Z}_{\mathrm{zd}}=4.0 \Omega$.

(1) Breaking fault of PT and fault lifting

Figure 4 is a disconnection fault and 0.085 disconnection fault simulation results released voltage transformer occur in 0.045 . As can be seen from Figure 4 (b), whether or disconnection fault disconnection fault lift, conventional criterion can be quickly identified.

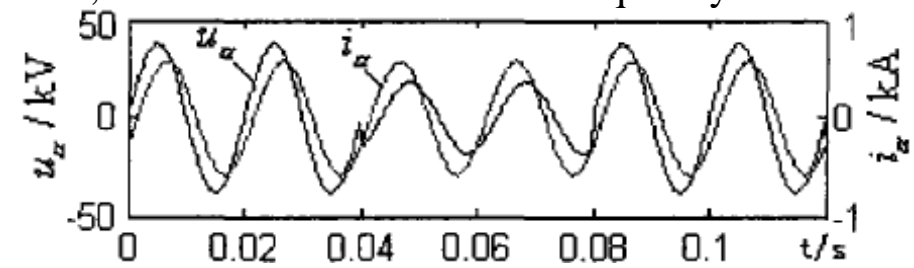

(a) voltage and current waveforms

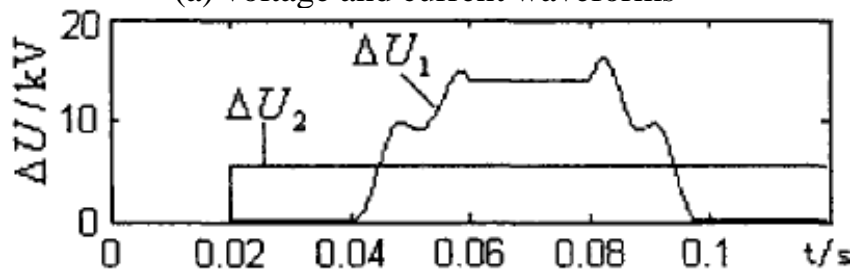

(b) judgment waveforms of breaking fault

(2) Effect of load varying to judgment

Fig.4 breaking fault and fault lifting

Sudden short-circuit fault in no load operation would lead to maximum load variety. Figure 5 shows the conventional criterion in this case the simulation results. Easy to know from Figure 5, the conventional criterion does not lead to changes in load misjudgment.
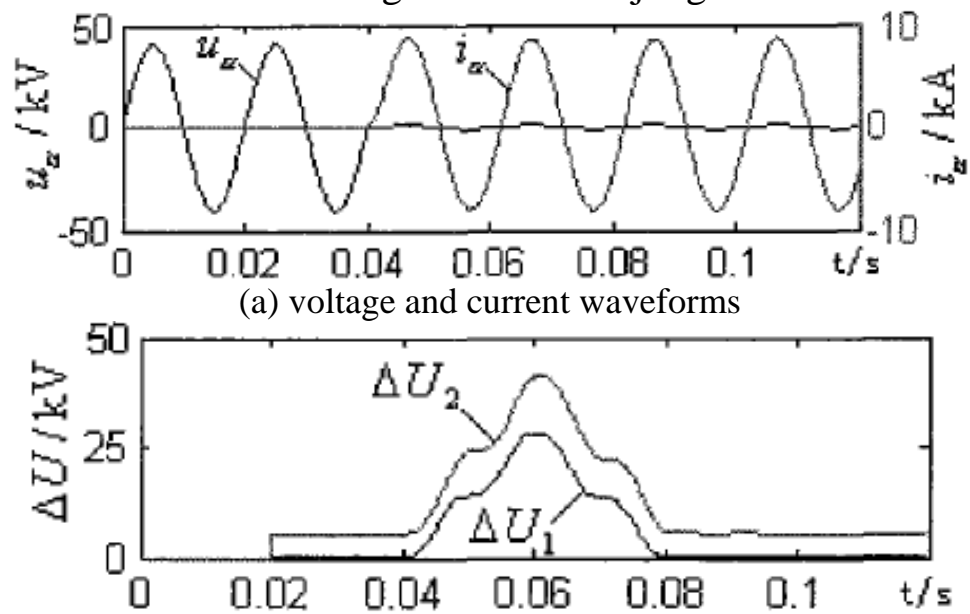

(b) judgment waveforms of breaking fault

Fig.5 Effect of load varying to judgment

(3)Effect of system impedance variety to judgment

With the power system operation mode between the maximum and minimum operation mode changes, the system impedance also changes. Figure 6 is a mutation of the power system simulation results for the maximum operating mode when the minimum operating mode. Figure 6 shows that the change does not affect the system impedance of the conventional criterion of validity. 


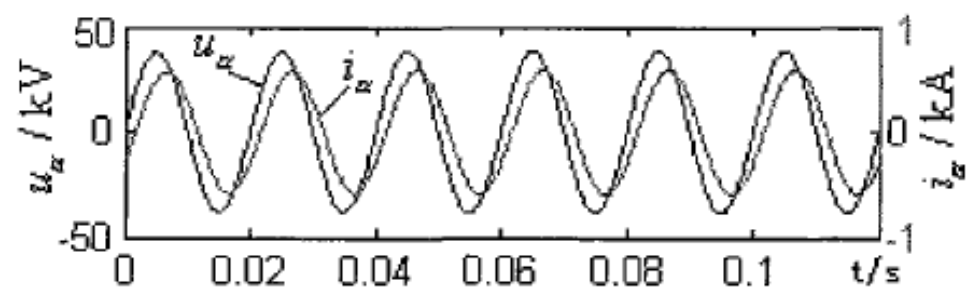

(a) voltage and current waveforms

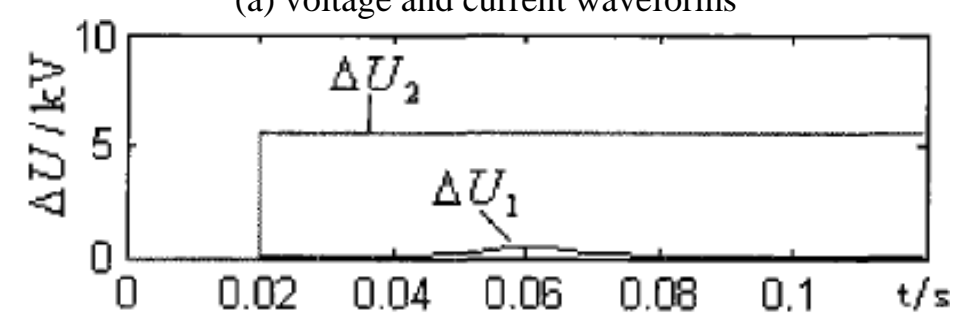

(b) judgment waveforms of breaking fault

Fig.6 Effect of system impedance variety to judgment

\section{Conclusions}

Substation using "three-instrument method" measuring secondly reactive power or high voltage fuse incomplete blowing, both will make protection devices measure high voltage when breaking fault occurs. It makes identification of PT breaking fault difficult. In this paper, these two cases are principally analyzed. After that, applicability of conventional criterion is taken simulation verification in changing of load and impedance. As a result, it will not be affected.

\section{References}

[1] H. Yokota,K. Chowdhury,R. Koodli,B. Patil,F. Xia.Fast Handovers for Proxy Mobile IPv6. draft-ietf-mipshop-pfmipv6-11 . 2009

[2]J.Sa.Silva, TCamilo,ACosta,et al.Exploring network mobil-ityin IPv6environments—issues and lessons learnt. IEEE Int'l Conf on Systems,Man and Cybernetics . 2004

[3] K.Lee,J. Park,H. Kim."Route optimization for mobile nodes in mobile network based on pr?ex delegation,". IEEE 58th Vehicular Technology Conference . 2003

[4] A.Patel,K.Lenng,M.Khalil.Mobile Node Identifier Option for Mobile IPv6(MIPv6). RFC4283 . 2005

[5] D. Johnson,C. Perkins,J. Arkko.Mobility Support in IPv6. RFC 3775 . 2003 